\title{
El enfoque jerárquico en el núcleo de los planteamientos organicistas y sistémicos en biología
}

\section{The hierarchical approach in the organicist and systemic positions in biology}

\author{
JON UMEREZ \\ IAS-Research - Philosophy of Biology group \\ Depto. de Lógica y Filosofía de la Ciencia \\ Universidad del País Vasco (UPV/EHU)
}

\section{RESUMEN}

Si los elementos conceptuales que caracterizan el organicismo del siglo XX son la centralidad del organismo, la perspectiva sistémica y el pensamiento jerárquico en términos de niveles de organización, planteo que el enfoque jerárquico es la «marca» de una perspectiva propia o genuinamente sistémica. De forma complementaria, defiendo que la ausencia de un enfoque genuinamente jerárquico en algunas propuestas actuales, por ejemplo, en el campo de la emergente biología de sistemas, es indicativo del diferente origen y ambición de tales planteamientos. Como ilustración específica de este planteamiento presento la larga trayectoria del trabajo experimental y teórico de Paul A. Weiss.

\section{PALABRAS CLAVE}

BIOLOGÍA SISTÉMICA, JERARQUÍA, ORGANICISMO, PAUL A. WEISS

\section{ABSTRACT}

If the conceptual traits that characterize XXth century organicism are centrality of the organism, systemic perspective, and hierarchical thinking in terms of levels of organization, I maintain 
that hierarchical thinking is the «mark» of a properly or genuinely systemic perspective. Complementarily, I claim that the absence of a genuinely hierarchical approach in some current proposals as, for instance, within the emerging area of systems biology reveals the diverse origin and ambition of those positions. As a specific example I present the long trajectory of experimental and theoretical work of Paul A. Weiss.

KEYWORDS

SYSTEMS BIOLOGY, HIERARCHY, ORGANICISM, PAUL A. WEISS

\section{INTRODUCCIÓN}

HAY AL MENOS TRES AMPLIOS ELEMENTOS CONCEPTUALES que caracterizan, de modo bastante patente, el significado filosófico de las corrientes organicistas vinculadas a la biología del desarrollo de principios del siglo XX: centralidad del organismo, perspectiva sistémica y pensamiento jerárquico en términos de niveles de organización.

La hipótesis que pretendo explorar en este trabajo mantiene que uno de los aspectos cuya fortuna merece ser escrutada, desde un punto de vista epistemológico, como señal de la persistencia del Organicismo (y la Biología Teórica) de principios del siglo XX en décadas posteriores, es, precisamente, el pensamiento jerárquico pues se da la circunstancia de que tanto su presencia como su olvido coinciden con una actitud más general (favorable o contraria, respectivamente) con el organicismo en las ciencias de la vida. Incluso aunque esta visión jerárquica sea, en algunos casos, el único indicio aparente de la visión más abarcadora. Propongo afianzar la hipótesis analizando el caso de Paul Weiss como muestra representativa de esta perspectiva.

De forma complementaria, planteo que la ausencia de un enfoque genuinamente jerárquico en algunas propuestas actuales, por ejemplo, en el campo de la emergente biología de sistemas es indicativo del diferente origen y ambición de tales planteamientos.

\section{ORGANISMO, ORGANIZACIÓN Y JERARQUÍA}

Este trabajo se inserta en el marco de la investigación acerca de la presencia y evolución de la biología teórica de corte organicista a lo largo de siglo XX, formulada e iniciada en un trabajo previo publicado en Ludus Vitalis (Etxeberria \& Umerez 2006).

Ahí mantenemos que la cuestión acerca de la organización biológica ocupa un lugar central entre los problemas teóricos a los que se enfrenta la Biología de la primera mitad del siglo XX. Una buena parte de las tradiciones que constituyen el ámbito de la Biología Teórica de esa época se ocupan de clarificar los 
fundamentos conceptuales de la disciplina, intentando dar respuesta al desafío (de raigambre kantiana) de la organización autónoma y proporcionar las herramientas metodológicas más apropiadas para la tarea. El organicismo tiene su más pleno desarrollo en este contexto con el propósito de superar definitivamente la prolongada y estéril oposición entre vitalismo y mecanicismo (clásico).

En el citado artículo describimos asimismo el progresivo desvanecimiento que el propio concepto de organismo experimentó a lo largo del siglo XX, desde su difíciles inicios a principios del mismo (Woodger 1929, p. 6), pasando por resultar cada vez más prescindible a medida que se asienta el auge de la biología molecular hasta su práctica desaparición a finales tal y como denuncian ciertos autores (Etxeberria \& Umerez 2006, pp. 5-7).

La hipótesis que exploro aquí se sustenta en ese trabajo y lo complementa iniciando el estudio de las raíces organicistas del pensamiento jerárquico que, habiendo estado claramente presentes en el organicismo y la Biología Teórica de principios del siglo XX, reaparece con bastante intensidad, aunque limitada, entre los años 60 y 70 de ese siglo y vuelve a adquirir importancia en la actualidad. Es decir, si nos centramos en estos aspectos (concepto de organización biológica y perspectiva jerárquica), podemos identificar también los tres períodos que distinguíamos en el devenir de la Biología Teórica del siglo pasado (Etxeberria \& Umerez 2006) aunque con diferentes proyección y limitaciones y cada uno con sus diversas propuestas teóricas y ámbitos disciplinarios más receptivos: en el primer período hasta la II Guerra Mundial, las teorías de la Evolución Emergente y de los Niveles de Integración, el «Theoretical Biology Club» y el embrión de la Ciencia de Sistemas, junto al desarrollo de la morfología y la embriología; en el segundo período, entre los años 60 y 70, las teorías de la autoorganización y los modelos abstractos y computacionales, junto a la Biología del desarrollo; en el tercero y actual, potencialmente, la Biología de Sistemas (Systems Biology) junto a la Biología evolutiva del desarrollo (Evolutionary Developmental Biology o Evo-Devo) o diversas áreas de la Epigenética.

Por otro lado, si nos centramos en autores concretos, la reflexión acerca del concepto de nivel de organización constituyó un elemento clave en las teorías de figuras relevantes en la puesta en marcha de este enfoque como Needham, Novikoff, von Bertalanffy, Woodger y otros. Asimismo, los autores responsables del resurgimiento, a contrapelo, en las últimas décadas del siglo pasado (Polanyi, Simon, Pattee, entre otros) mantienen o retoman la preocupación por este concepto y, en particular, intentan dar cuenta del papel de la relación internivélica en la organización de los sistemas biológicos. En la tercera etapa, sin embargo, no es tan claro que las líneas de investigación actuales confieran al elemento jerárquico un significado igualmente relevante. 


\section{Paul A. Weiss}

Entre esos autores, el embriólogo y neurólogo Paul Alfred Weiss representa de forma muy apropiada la continuidad de este punto de vista, desde el principio de su carrera en los años 20 hasta su intensa actividad en los años 60 y 70.

\section{1. NOTAS BIOGRÁFICAS}

Weiss nació en Viena el 21 de marzo de 1898 en una familia acomodada y en un entorno cultivado (murió el 8 de septiembre de 1989). Durante la Gran Guerra sirvió tres años como oficial de artillería y, al terminar ésta, el mismo otoño de 1918, comenzó Ingeniería Mecánica en la Technische Hochschule (actualmente Technische Universität Wien) aunque al año siguiente se pasó a Biología en la Universität Wien (Brauckmann 2003, 2004).

Inmediatamente, en 1920, logra un puesto para trabajar en los laboratorios del Biologische Versuchsanstalt, el ya reputado Prater Vivarium (a causa del lugar de la ciudad en el que se sitúa). El Instituto se inaugura a principios de siglo y es fruto del esfuerzo y la aspiración para emular otros centros de investigación experimental en el mundo. Su director es Hans Przibram, fundador del mismo junto a Leopold von Portheim y Wilhelm Fiador, y entre sus integrantes más conocidos figura también, por ejemplo, Paul Kammerer (ver también Drack \& Apfalter 2007 y Drack et al 2007 para más información). ${ }^{1}$

Es ahí donde Weiss concluye una serie de estudios experimentales para su tesis doctoral (1922), bajo la dirección del propio Przibram, acerca de las posiciones de descanso de las mariposas en respuesta a condiciones de luz y gravedad centrados en sus alas, y varias publicaciones, en particular un largo artículo (Weiss 1925) cuya versión en inglés fue publicada años después, precisamente, en el General Systems Yearbook of the Society for General System Research (Weiss 1959).

Cuando Kammerer se retira, en 1924, Weiss accede al puesto de Adjunkt (única posición remunerada en el Instituto) y permanece en él hasta 1927 cuando obtiene una beca de la Fundación Rockefeller y, con una excedencia, viaje a diversos países para llevar a cabo su trabajo de investigación acerca del cultivo

1 No conozco en la literatura, ni he explorado más allá, datos acerca de la posible relación intelectual entre Weiss y Kammerer durante ese breve período en el que coinciden en el Vivarium, al contrario, por ejemplo de lo que sucede con von Bertalanffy, cuya relación de vecindad y amistad entre las familias cuando era joven, es sabida (Drack \& Apfalter 2007, Drack et al 2007). No obstante, esa conexión tiene una segunda parte, bien que indirecta, en el contexto de las relaciones posteriores de Weiss con Koestler (y su participación en la conferencia de Alpbach que mencionaremos a continuación), cuyo intento de vindicación de Kammerer (no alejado por cierto en sus fundamentos del objetivo de la citada conferencia) tuvo tanto eco en la época (Koestler 1971). 
de tejidos y la re-enervación nerviosa y muscular en el Instituto Oceanográfico de Mónaco, en la Sorbona en París y en el Kaiser-Wilhelm-Institute for Biology en Berlin-Dahlem. En 1929 renuncia definitivamente a esa posición pero, al año siguiente, el puesto que tenía en perspectiva en la Universidad de Frankfurt no se materializa, como consecuencia de los efectos de la depresión económica, y entonces Weiss se marcha a EE.UU. (ver Brauckmann 2003).

Allí, en 1931 gana una beca Sterling para trabajar con Ross Granville Harrison en Yale y ese septiembre comienza su carrera estadounidense (ver Brauckmann 2003). Posteriormente, en 1934, consigue un puesto como profesor titular (Assistant Professor) en la Universidad de Chicago y ya en 1942 obtiene la cátedra de Zoología (Professor) en esa misma universidad. Permanecerá en Chicago hasta que, en 1954, el Instituto Rockefeller le ofrece incorporarse al mismo en calidad de Director del recientemente fundado Laboratorio de Biología del Desarrollo (Laboratory of Developmental Biology). Weiss se establece en New York hasta su jubilación con algunas breves excepciones, p. ej. como profesor visitante de Biología en el MIT en 1956 o su frustrada experiencia como decano (Dean) de la Graduate School of Biomedical Sciences en la Universidad de Houston (TX) entre 1965 y 1966 (Brauckmann 2003).

\section{III.2. INFLUENCIA}

La labor investigadora realizada por Weiss a lo largo de más de 5 décadas (sufrió un ataque cerebral en 1978 del que no se recuperó) tuvo un indiscutible impacto entre sus contemporáneos. Su trabajo en morfogénesis y diferenciación celular junto a su versión original de la teoría del campo morfogenético influye principalmente en algunas áreas de la biología como la biología del desarrollo, la biología celular y la neurobiología. Su reflexión teórica más general también tiene eco, aunque hasta su etapa final no se ocupa tanto de aspectos epistemológicos generales de forma explícita (a pesar de mantener su pronunciada prevención ante la caracterización filosófica o teórica de su pensamiento: insistencia en su neutralidad filosófica y en su estricta derivación de la práctica experimental).

En un aspecto de orden más administrativo y de carácter, por lo tanto, más superficial pero que refleja el trasfondo de su enfoque sistémico y jerárquico, el propio Weiss menciona que fue él quien estableció la clasificación, precisamente jerárquica, de las ciencias biológicas que posteriormente deviene estándar:

On assuming the chaimanship of the Division of Biology and Agriculture of the National Research Council in 1951, I reestructured the administrative subcategorization of «biology», previously based on forms of life (botany, zoology, bacteriology, etc.) or on methods of study (anatomy, biochemistry, biometrics, etc.), by a hierarchical system of order according to functional principles in common to 
living organisms: Molecular, Cellular, Genetic, Developmental, Regulatory, and Group and Environmental Biology. (Weiss 1969, p.10 n.2)

De forma más precisa y cuantitativa se podría indicar que, tal y como señala Brauckman (en tiempos pre-Web of Science o pre-JCR), apareció reseñado como uno de los 250 primeros autores más citados para el período 1961-1975 (Garfield 1977, citado en Brauckmann 2003, 5). Asimismo, a lo largo de su carrera, fue merecedor de numerosos premios, títulos honoríficos y otros reconocimientos, entre ellos la National Medal of Science, otorgada por Carter en 1979. A pesar de esos éxitos, al final Sabine Brauckman se pregunta:

Recalling Weiss' contributions to the biological sciences and the many awards and honorary degrees he received, it is astonishing that historiography of biology has barely recognized the role that Weiss played in American biology of the 20th century. About the reasons one might speculate [...] (Brauckmann 2003, 5).

Tal vez esas razones sobre las que sólo podemos especular tengan que ver con el propio «purgatorio» de los enfoques sistémicos durante gran parte del siglo XX. No obstante, algo más de 30 años antes, en cierto sentido en la cima de su influencia (teórica, «filosófica»), June Goodfield puso a Weiss como ejemplo de «buen» anti-reduccionista:

And amongst the antireductionists of the twentieth century we can find one example of a biologist whose ideas stand out as brightly coloured threads in the fabric of theoretical and philosophical biology. For, as a first-class experimentalist, whose laboratory 'hardware' both in terms of empirical results and conceptual innovation is widely recognized and appreciated, Paul Weiss stands apart from the majority of antireductionists in the organismic school of biology. For above all he is an experimentalist, whose philosophical statements have been backed up by a wealth of empirical detail, [...] (Goodfield 1974, 73)

Según Goodfield, Weiss merece esa distinción, precisamente, a causa de su trabajo experimental. Así que volvamos un momento a él. En cualquier caso, a continuación, habremos de situar también a Weiss en el contexto de los debates teóricos, filosóficos, de finales de los 60 y principios de los 70 , en el que en mi opinión encontramos otra de las claves de esa apreciación contemporánea, enunciada justo en esa época «de posibilidades» que detectamos en nuestro trabajo previamente citado (Etxeberria \& Umerez 2006), así como, probablemente, algunas pistas acerca de su relativo olvido posterior, sobre las que podemos especular en compañía de Brauckman. 


\section{III.3. TROPISMOS ANIMALES DE LOEB}

A partir de la publicación en 1912 de The Mechanistic conception of Life, Loeb se pasa al estudio experimental de los tropismos animales como base para el estudio del libre albedrío. ${ }^{2}$ Resultado de esas indagaciones es el libro sobre Forced movements, Tropisms, and Animal Conduct, publicado en 1918, donde comienza con fenómenos familiares como los de las plantas orientándose con respecto a la luz y la gravedad en su crecimiento, sigue con comportamientos similares en organismos sésiles y termina por abordar los movimientos aparentemente condicionados por factores externos que se dan en animales con capacidad de movimiento autónomo.

Recordando más adelante esos trabajos, Weiss presenta la propuesta de Loeb como el epitome del enfoque mecanicista que se propone combatir:

The explanation by Jacques Loeb (1918) of animal behavior in terms of rigidly concatenated reflex sequences, and particularly his proposition of tropisms as paradigms of a precise cause-effect machine principle in organisms, epitomizes that kind of mechanistic preconception. (Weiss 1969,74)

\section{III.4. EXPERIMENTOS DE WEISS}

En concreto, los experimentos que realiza para su tesis doctoral son diseñados para determinar si y hasta qué punto las posturas normales de las mariposas están relacionadas con la dirección y la intensidad de la luz y con la gravedad. Para investigar esos comportamientos y determinar los factores que los provocan procede metódicamente de la siguiente manera: por un lado, establece una o dos fuentes de luz y, por otro, introduce cambios en el ángulo de inclinación, de vertical a más horizontal, de la pared sobre la que se posan. Este estudio sistemático de la influencia de los factores externos permite no sólo calibrar su respectiva preponderancia sino también atisbar hasta qué punto hay un núcleo en esos comportamientos (y otros similares) que no depende de condicionantes externos (ver Drack et al 2007).

En el curso de los experimentos, primero investiga la influencia de los factores individuales por separado y después en combinación. Para centrarse en la influencia de la luz, iguala el aspecto gravitacional permitiendo a las mariposas únicamente asirse al techo y modifica paulatinamente la dirección de la luz. Complementariamente, para medir la influencia de la gravedad deja

2 Los tropismos son los movimientos permanentes de la planta o de algún órgano, como respuesta a un estímulo externo que actúa en una sola dirección, de modo que la planta crece hacia el estímulo (Wikipedia) 
fija la iluminación y permite a las mariposas posarse en la pared procediendo a cambiar gradualmente el ángulo de inclinación de la misma desde la posición vertical hasta suspenderla horizontalmente. Los experimentos se completan combinando diversas fuentes de luz y analizando el efecto de ambos factores en conjunción.

Aunque demuestra que tales factores (luz y gravedad) ejercen su influencia, lo que queda de manifiesto en los experimentos en los que sólo uno es alterado, los resultados establecen que la posición de las mariposas no se puede predecir con exactitud a partir del conocimiento de la dirección de la luz y la gravedad, sino que de la posición de descanso que las mariposas van a adoptar finalmente sólo se puede proporcionar una estimación aproximada. Según sus propias palabras, los experimentos confirmaron sus conclusiones teóricas de corte sistémico:

[...] the experiments totally confirmed theoretical conclusions. It could be demonstrated that knowing the efficacy of single factors within a complex allowed unequivocal conclusions to be drawn about the efficacy of the entirety from which we previously isolated single parts; all positions [of the butterflies] turned out to be demonstrable as coming off the entirety of those partial effects, an exclusive efficacy of one single factor was never observed (Weiss 1922, 19, citado en Drack et al 2007).

Es más, Weiss da a entender que habría que tener en cuenta una especie de memoria interna, dado que la experiencia previa parece jugar un papel importante. En general, aunque en otros términos, pone de manifiesto la relevancia de la dinámica interna y los procesos de auto-organización presentes con sus capacidades de equilibrio y compensación.

Como resultado de su investigación, Weiss ofreció una sólida crítica a la teoría mecanicista de los tropismos de Loeb, mostrando que se basaba en asumir que la similitud de movimientos en diferentes casos tenía que ser debida a una identidad en hipotéticos mecanismos subyacentes, y ofrecía alternativamente «una teoría general de sistemas del comportamiento animal» (ref. Weiss 1969) que implicaba un explícito enfoque jerárquico.

Weiss señala en esos trabajos $(1922,1925)$ que la dinámica interna es más compleja y diversa. En suma, su famosa afirmación de que «los organismos no son marionetas operadas por los hilos del entorno» («organisms are not puppets operated by environmental strings»):

[...] the beginning and the end of a behavioral act could often be unequivocally correlated with a cue from the environment, but the execution of the given act 
was found to be so variable and indeed unique in detail, from case to case and from instance to instance that it was gratuitous to maintain that the attainment of essentially the same result regardless of the variety of approaches is simply the blind outcome of a chain of seriated steps appropriately pre-designed by evolution to lead to that end. (Weiss 1969,4 ).

Igualmente, en esos años, Weiss desarrolla su trabajo en biología del desarrollo en diversos ámbitos, tanto estudios clásicos como los de regeneración y trasplantes en vertebrados o mas propios como los relativos al desarrollo de las células nerviosas. Prosigue también la elaboración su teoría de campo (field theory) para el desarrollo ontogenético.

\section{ConTEXTO MÁS AMPLIO EN BIOLOGÍA}

Tanto el pensamiento jerárquico en particular como la propia situación de Weiss en el curso de su larga trayectoria científica, sufrirán la influencia de esos tres periodos que mencionaba en el apartado 2 y que hemos formulado en Etxeberria \& Umerez (2006). A este respecto, el contexto mas relevante es el que tiene que ver, por un lado, con el relativamente rápido surgimiento e implantación de la biología molecular centrado en torno a la elucidación de la estructura del ADN y su papel en la síntesis proteínica (de 1953 a 1966) y, por otro lado, con la más prolongada historia de la paulatina transformación de la biología evolutiva, centrada a su vez en el eje de la formulación de la Síntesis Moderna (de 1936 a 1947, según la delimitación canónica de Mayr \& Provine 1980).

En ambas áreas, nos encontramos con una situación más abierta y, por decirlo así, menos dogmática en el período de entreguerras en el que Weiss inicia y asienta su carrera científica. A partir de los años 50, sin embargo, se afianza en ambos casos una visión ortodoxa unificada (seleccionismo, adaptacionismo y extrapolacionismo micro-macroevolutivo junto a reducción de la función orgánica a estructura molecular) que no deja mucho margen para concepciones diversas, por ejemplo, las más sistémicas provenientes del trabajo en biología del desarrollo. No obstante, como ya he mencionado, tanto por el trabajo en esas áreas menos sujetas a las ortodoxias neodarwinista y molecular estrictas y que logran exponer sus limitaciones como por motivos de cariz interno (por ejemplo, respectivamente, las cuestiones del neutralismo o la regulación), en los años a caballo del cambio de década de los 60 a los 70 del sigo pasado se abren de nuevo posibilidades para discutir en círculos más amplio enfoques que incorporan elementos organicistas, sistémicos, auto-organizativos y no reduccionistas. Este paréntesis se cerrará pronto y la ortodoxia precedente saldrá incluso reforzada temporalmente hasta la situación más actual que estamos intentando evaluar (para más detalles, ver Etxeberria \& Umerez 2006, pp. 10-15). 


\section{Participación de Weiss en los debates POSTERIORES}

La perspectiva sistémica va a seguir siendo fundamental en su posterior trabajo científico (embriología, neurología, biología celular en general, etc.) y va a ser central en su contribución a los debates sobre el lugar de la biología a finales de los años 60 y principios de los años 70. Esta participación se queda bien ejemplificada en el libro que editó acerca de los Hierarchically Organized Systems in Theory and Practice (Weiss 1971b) donde se incluye su ensayo centrado en las ciencias biológicas «The Basic Concept of Hierarchic Systems» (Weiss 1971a) que recoge la introducción a la edición revisada y actualizada de su libro clásico acerca de la dinámica del desarrollo biológico (Weiss 1968), apelando al enfoque sistémico:

Conceptually the life sciences are entering a phase of maturation. Yet, time has come when growing knowledge is forcing them to cease acquiescing in noncommittal symbolic cover terms for properties of living systems such as «growth», «organization», «specificity», «metabolism», etc., and to go on to describe precisely and without equivocation the factual content of the phenomena thus labeled. And if one pursues this course judiciously [...] one comes to the realization that the trend from naïve microdeterministic causality toward macrodeterministic «system» theory is inexorable (Weiss 1971, vii).

También hay que destacar, entre otras aportaciones, trabajos teóricos como el famoso $\ll 1+1 \neq 2 »$ (Weiss 1967), con su amplio repertorio de ejemplos empíricos; la extensa y profunda contribución al Simposio que organizó Arthur Koestler en Alpbach, Beyond Reductionism, titulada «The living system: determinism stratified» (Weiss 1969); ${ }^{3}$ o el más divulgativo «The System of Nature and the Nature of Systems: Empirical Holism and Practical Reductionism Harmonized» (Weiss 1977); así como su libro de reflexión acerca de las ciencias de la vida (Weiss 1973). En todos estos trabajos, así como en su carrera como científico y teórico en las décadas anteriores, Weiss practica y defiende con rigor y pro-

3 En el volumen resultante (Koestler \& Smythies 1968), además de la de Weiss, se publicaron las contribuciones de L. von Bertalanffy, J. S. Bruner, V. Frankl, F.A. Hayek, H. Hyden, S. S. Kety, A. Koestler, P. D. MacLean, D. McNeill, J. Piaget junto a B. Inhelder, J.R. Smythies, W.H. Thorpe y C.H. Waddington. Además de subrayar la presencia Piaget y Waddington o el propio von Bertalanffy hay bastantes aspectos interesantes, que no puedo estudiar aquí, en este grupo de Alpbach que, según Haraway, forma una comunidad «paradigmática» (paradigm community) conocida como el grupo Frensham (Frensham group), patrocinado por la Fundación van Leer (Haraway 1975, p. 149, p. 184). 
fundidad una perspectiva que considero que puede ser de utilidad para abordar cuestiones y debates abiertos de nuevo en la actualidad.

En este sentido, se puede apuntar brevemente que además de desarrollar los conceptos claves de este enfoque como los propios de sistema, organización, jerarquía o constricción, fruto de su interés por la epistemología y la teoría del conocimiento, desarrolla también una dialéctica entre abstracción analítica y reconstrucción sintética en términos de pérdida dialéctica de detalle e información. Asimismo, a raíz de esas reflexiones adopta posiciones complejas en términos de complementariedad acerca de la dicotomía atomismo/holismo y propone una idea de determinismo estratificado sin dejar de insistir en la génesis de la organización a partir de la organización. Por último, me gustaría mencionar que elabora también su claro posicionamiento contra el determinismo genético $\mathrm{y}$, en concreto, su escepticismo con respecto al (mal)uso de «información» $\mathrm{y}$, principalmente del lenguaje asociado (con verbos o acciones); así como su crítica de esos conceptos en tanto que forma de antropomorfismo y, consecuentemente, como forma de rehuir la cuestión.

La elección de Weiss como hilo conductor de esta exploración se basa en la continuidad personal que establece su trayectoria de más de 50 años (a diferencia por ejemplo de Needham) y en su trabajo experimental en los campos de la embriología y la neurología, lo que hace del suyo un caso excepcional como ha explicado Haraway en detalle (1976) o como indica June Goodfield en su más que elogiosa apreciación previamente citada.

\section{CONCLUSIONES}

Volviendo a la tesis del marco conceptual (Exeberria \& Umerez 2006) en el que se enmarca este trabajo, mantenemos las constatación de que en estos últimos años se vuelven a abrir, al menos potencialmente, nuevas oportunidades para los enfoques de corte organicista y sistémico. Señalábamos allí que esta reapertura se podía deber a la coexistencia de dos factores: por un lado, el propio éxito de la biología molecular que pone al descubierto sus limitaciones explicativas (expresión, regulación) y, por otro, la prudentemente exitosa irrupción de enfoques renovados como los relacionados con las llamadas ciencias de la complejidad y de sub-disciplinas con capacidad de ofrecer innovaciones teóricas y resultados empíricos como la Evo-Devo o los estudios de sistemas auto-organizativos. Todo ello unido al desarrollo, tanto desde enfoques tradicionales como alternativos, de herramientas formales y computacionales de gran capacidad que permiten superar los desafíos del reduccionismo (Gilbert \& Sarkar 2000)

Así, podemos constatar que, por ejemplo, un número especial en Science dedicado a la Biología de Sistemas tiene por título genérico «Whole-istic Biology» (Chong \& Ray 2002) y contiene explícitamente, por ejemplo, referencias a ideas de las ciencias de la complejidad o a von Bertalanffy, aunque paradóji- 
camente no a sus trabajos más propiamente biológicos (sus monografías de los años 30 y principios de los 40) sino a su posterior reformulación en el contexto de la Ciencia de Sistemas.

Esto nos muestra, simultáneamente, la apertura de opciones de recuperar a determinados precursores y al vez, la necesidad de hacerlo genuinamente para evitar deformaciones. En este sentido, no faltan autores de reconocida trayectoria que comienzan a mostrar su preocupación por el riesgo de cooptación de la Biología de Sistemas por parte de enfoques sin vinculación o compromiso con los aspectos más organicistas y propiamente complejos que son, por contra, poco mas que ajustes del anterior paradigma molecular predominante. Así, alguien como Woese, tras largos años trabajando acerca de la complejidad de código genético y revolucionando la microbiología o la más moderna versión de la estructura del «árbol de la vida» entre otros temas, se lamenta de enfoque ingenieril (engineering approach) que se le está dando mayoritariamente a la Biología de Sistemas. O Mesarovic, co-autor de uno de los libros acerca de la estructura de los sistemas jerárquicos en los años 70 , evidencia precisamente la cortedad de miras de una perspectiva carente de niveles propiamente (flat earth perspective) (Mesarovic \& Sreenath 2006). En un sentido más amplio y conceptual (O’Malley \& Dupré 2005) reclaman la necesidad de hacer ulteriores esfuerzos filosóficos a la ahora de abordad los aspectos fundamentales de la disciplina en ciernes y Kirschner (2005) señala en particular la necesidad de incluir aspectos contextuales e históricos que permanecen por lo general ausentes. Stuart Newman por su parte denuncia el riesgo patente de una vuelta a la más ramplona simplificación (Newman 2003).

En consecuencia, a partir de esos trabajos de Weiss y considerando el contexto conceptual en las diferentes épocas, he abordado un análisis prelimar acerca de la conexión entre su trabajo científico experimental en cuestiones, sobre todo, de biología del desarrollo y su perspectiva teórica fundada en una concepción explícitamente sistémica y jerárquica, como una forma de evaluar el alcance y los límites de su influencia, así como de la potencialidad de su enfoque (y la de otros científicos con una perspectiva teórica similar) para abordar los problemas de las ciencias biológicas en diferentes momentos del siglo pasado y hoy en día.

El análisis de la aportación de Weiss corrobora que un enfoque jerárquico es la «marca» de una perspectiva propia o genuinamente sistémica en contraste con tendencias contemporáneas que constituyen más bien formas de cooptación formal pero no sustantiva. 


\section{Agradecimientos}

El autor agradece la financiación del Proyecto FFI2011-25665, Ministerio de Economía y Competitividad y de la UE (Feder), y de la Subvención a Grupos IT 505-10, Gobierno Vasco.

\section{REFERENCIAS BIBLIOGRÁFICAS}

BRAUCKMANN, Sabine (2003) «The Scientific Life of Paul A. Weiss (1898--1989)». Mendel Newletter 12: 2-7. , (2004) «Paul A. Weiss, 1898-1989: The Cell Engineer». In Darwin H. Stapleton (ed.) Creating a Tradition of Biomedical Research: Contributions to the History of the Rockefeller University. New York: Rockefeller University Press, pp.: 271282.

CHONG, L., RAY, L.B. (2002) «Whole-istic biology». Science 295: 1661.

DRACK, Manfred \& APFALTER, Wilfried (2007) «Is Paul A. Weiss' and Ludwig von Bertalanffy's System Thinking Still valid Today». Systems Research and Behavioral Science 24: 537-546.

DRACK, Manfred, APFALTER, Wilfried \& POUVREAU, David (2007) «On the making of system theory of life: Paul A. Weiss and Ludwig von Bertalanffy's conceptual connection». The Quarterly Review of Biology 82(4): 349-373.

ETXEBERRIA, Arantza \& UMEREZ, Jon (2006) «Organismo y Organización en la Biología Teórica ¿Vuelta al organicismo?» Ludus Vitalis XIV(26): 3-38.

GARDFIELD, E. (1977) The 250 «Most-Cited primary Authors, 1961-1975». Part II. The Correlation Between Citedness, Noble Prizes and Academy Memberships. Current Comments 50: 5-15.

GILBERT, S. F. \& SARKAR, S. (2000) «Embracing complexity: Organicism for the 21st Century», Developmental Dynamics 219: 1-9.

GOODFIELD, June (1974) «Changing Strategies: A comparison of Reductionist Attitudes in Biological and medical research in the Nineteenth and Twentieth Centuries». In F.J. Ayala \& Th. Dobzhansky (eds.) Studies in the Philosophy of Biology. Reduction and Related Problems, Berkeley, CA: University of California Press, pp. 65-86 (73-86).

HARAWAY, Donna J. (1976) Crystals, Fabrics, and Fields. Metaphors of Organicism in Twentieth-Century Developmental Biology. Newhaven, CT: Yale University Press (reprinted 2004 Berkeley, CA: North Atlantic Books, foreword by S.F. Gilbert)

KIRSCHNER, M.V. (2005) «The meaning of systems biology». Cell 121 (4): 503504.

KOESTLER, A. (1971) The Case of the Midwife Toad. New Cork: Random House. 
KOESTLER, A. \& SMYTHIES, J. R. (eds.) (1969 [1970]) Beyond Reductionism. New Perspectives in the Life Sciences (The Alpbach Symposium 1968). London: Hutchinson [New York: Macmillan]

LOEB, Jacques (1912) The mechanistic conception of life: biological essays, Chicago: The University of Chicago Press.

, (1918) Forced movements, tropisms, and animal conduct. Philadelphia: Lippincott.

MAYR, E. (1980) «Prologue: Some Thoughts on the History of the Evolutionary Synthesis». En Mayr \& Provine (eds.), pags. 1-48.

MAYR, E. \& PROVINE, W.B. (eds.) (1980) The Evolutionary Synthesis. Perspectives on the Unification of Biology. Cambridge, MA: Harvard University Press.

MESAROVIC, M., SREENATH, S.N. (2006) «Beyond the flat earth perspective in systems biology». Biological Theory 1(1): 33-34.

NEWMAN, S.A. (2003) «The fall and rise of systems biology». GeneWatch 16(4): 8-12.

O’MALLEY, M.A., DUPRÉ, J. (2005) «Fundamental issues in systems biology». BioEssays 27(12): 1270-1276.

OLBY, R. (1990) «The Molecular Revolution in Biology». En R. C. Olby, G.N. Cantor, J.R.R. Christie \& M. J.S. Hodge (eds.) Companion to the History of Modern Science, London: Routledge, pags. 503-520.

WEISS, Paul A. (1922) Richtungbestimmende Einflüse äußerer Faktoren. Die Ruhestellung der Vanssiden. Tesis doctoral, University of Vienna. , (1925) «Tierisches Verhalten als "SystemReaktion". Die Orientierung der Ruhestellungen von Schmetterlingen (Vanessa) gegen Licht und Schwerkraft». Biologia Generalis 1: 165-248.

,(1959) «Animal behavior as system reaction: orientation toward light and gravity in the resting postures of butterflies (Vanessa)». General Systems Yearbook of the Society for General System Research 4: 19-44.

, (1967) $\ll 1+1 \neq 2$ (One Plus One Does Not Equal Two) ». In G. Quarton, Th. Melnechuck \& F. Schmitt (eds.) The Neurosciences. A Study Program, New York: Rockefeller University Press, pp.: 801-821.

, (1968) Dynamics of Development. Experiments and Inferences. New York: Academic Press.

, (1969) «The living system: determinism stratified». In Koestler \& Smythies (eds.), pp. 3-55. , (1971) «The Basic Concept of Hierarchic Systems». In Weiss (ed.), pp. 1-43. , (ed.) (1971) Hierarchically organized systems in Theory and Practice. New York: Hafner Publishing.

, (1973) The science of life: The living system --a system for living. Mt. Kisco, NY: Futura Pub. Co.

,(1977) «The System of Nature and the nture of Systems: Empirical Holism and Practical Reductionism Harmonized». In K. E. Schaefer, H. Hensel \& R. Brady (eds.) A New Image of man in Medicine, Vol.I-Towards a Man-Centered Medical Science, Mt. Kisco, NY: Futura Pub. Co, pp.: 17-63. 
WOESE C.R. (2004) «A new biology for a new century». Microbiology and Molecular Biology Reviews 68(2): 173-186.

Jon Umerez es investigador-IAS en el Departamento de Lógica y Filosofía de la Ciencia de la Universidad del País Vasco.

Líneas de investigación:

Filosofía de la biología, Filosofía e Historia de la ciencia, sistemas complejos y Vida Artirficial, biología y sociedad, percepción social de la ciencia

Publicaciones recientes:

UMEREZ, J. (2009) «Where Does Pattee's "How Does a Molecule Become a Message?" Belong in the History of Biosemiotics». Biosemiotics 2(3): 269-290 (answer in Pattee, H.H. (2009) «Response by H.H. Pattee to Jon Umerez's paper: "Where Does ...." ». Biosemiotics 2(3): 291-302).

RUIZ MIRAZO, K.; UMEREZ, J. \& MORENO, A. (2008) «Enabling conditions for 'open-ended evolution'». Biology \& Philosophy 23(1): 67-85. Available online [0169-3867 (Print) 1572-8404 (Online) DOI 10.1007/s 10539-007-9076-8].

Dirección electrónica: jon.umerez@ehu.es http://www.ehu.es/ias-research/umerez/index_es.html 